\title{
THE EVOLUTION OF THE SUN'S ANGULAR MOMENTUM
}

\author{
DAVID R. SODERBLOM \\ Space Telescope Science Institute \\ 3700 San Martin Drive \\ Baltimore MD 21218 USA \\ JOHN R. STAUFFER \\ NASA-Ames Research Center \\ Moffett Field CA 94035 USA
}

\begin{abstract}
We discuss the observational data that are available to illustrate the rotational history of the Sun, and those data that are particularly pertinent to the interiors of the Sun and stars.
\end{abstract}

\section{Introduction}

Observationally, rotation is a purely surface phenomenon, yet there are aspects of its study in solar-type stars that have implications for the study of stellar interiors. This review will emphasize those aspects. For other recent reviews, see Hartmann and Noyes (1987) and Stauffer and Soderblom (1989). The reader is also referred to the forthcoming text by Giampapa and Sonett (1989) for discussions pertinent to solar rotation. Among the questions that are relevant to this meeting that rotation studies can address are:

Is the Sun typical for its mass and age?

Do planets play a role in the angular momentum history of the Sun?

What is the rotational history of a star like the Sun?

What surface phenomena are useful diagnostics of the Sun's interior?

Differential rotation.

Rotation and activity cycles.

Rotation and lithium depletion.

Is there stellar evidence for a rapidly rotating core in the Sun?

\section{The Sun Among the Stars}

Classical studies of stellar rotation using photographic plates reached their limit with the work of Kraft (1967), who demonstrated the connections between rotation, age, and chromospheric activity for stars like the Sun. Skumanich (1972) quantified Kraft's results in his $t^{-1 / 2}$ laws between rotation or activity and age. (Note that Skumanich based this on three data points.) Soderblom (1983) showed that the Sun has an average $v \sin i$ for an old solar-type star.

But the most important data for studying rotation in low mass stars has resulted from the

403

G. Berthomieu and M. Cribier (eds.), Inside the Sun, 403-413.

(C) 1990 Kluwer Academic Publishers. Printed in the Netherlands. 
intensive work on modulation of star light by stellar surface inhomogeneities, especially that done by the Mount Wilson group. They have now determined rotation periods for about 60 stars (Baliunas et al. 1983). These stars cover a broad range of age and mass, although there tend to be few old stars because the activity is so low in such stars, reducing chromospheric contrast to an unobservably low level. Thus there are not enough old solar-type stars to form a meaningful comparison sample. However, there are enough stars to calibrate a relationship between the level of chromospheric activity (quantified by $R_{\mathrm{HK}}^{\prime}$, the ratio of the star's Ca II H and $\mathrm{K}$ flux to its bolometric luminosity) and the Rossby number (the ratio of the rotation period to the convective turnover time). For details of this, see Noyes et al. (1984) and Gilliland (1987). Using such a relation, one or two observations of the HK flux are sufficient to determine the rotation period to an accuracy of $20 \%$ (Soderblom 1985).

If this relation is applied to the Vaughan and Preston (1980) survey of HK emission among solar-type stars, one derives the distribution of rotation rates for stars near $1 M_{\odot}$ (Fig. 1). For a discussion of this, see Soderblom (1985). The essential points are: (1) the Sun is quite typical for an old star of its color; (2) the range in rotation is about one dex at any one color (roughly the same mass), but most of the stars fall within a range of only a factor of 3 to 4 ; (3) the mass range represented is about 0.8 to $1.2 M_{\odot}$, and the distribution of stars here suggests that all that mass range has about the same relation between rotation and age; (4) for the redder stars, the minimum is $\sim 0.5 \Omega_{\odot}$, probably because at these low masses the evolutionary time scales exceed the age of the Galactic disk and these stars have not yet been able to evolve to very low velocities; and (5) the sharp bottom among the bluest stars is a selection effect because stars of those masses evolve away from the main sequence quickly, and are no longer included in a survey such as this (they are classified as

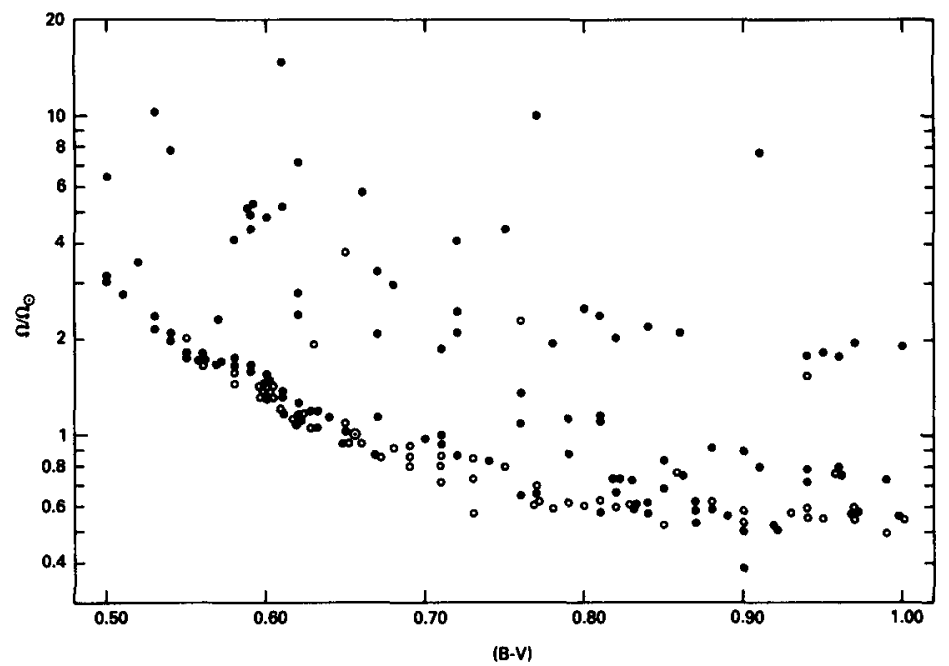

Figure 1. Predicted angular velocity (in solar units) versus $(B-V)$ color for solar-type stars of the Vaughan-Preston survey (from Soderblom 1985). The Sun is shown at $(B-V)$ $=0.656$ near the lower bound of the stars. Note that rotation appears to flatten out at about $0.5 \Omega_{\odot}$ for the lowest masses (reddest stars), and that the range at any one color is about one dex. The open circles represent high-velocity stars of the old disk population. 
subgiants instead of main sequence stars).

All of the above is consistent with the Sun having arrived on the main sequence rotating at about 4 times its present rate, and then having lost angular momentum by the $t^{-1 / 2}$ law, but the details are highly uncertain due to a lack of stars we can observe whose ages are known with confidence.

\section{A Brief History of Solar Rotation}

The field star studies outlined in the preceding section suggest that the rotational evolution of the Sun and other stars of similar mass stars is fairly sedate and well defined over most of their main sequence lifetimes. This suggests either that the star formation process enforces uniformity or that angular momentum evolution during the pre-main sequence and early main sequence period significantly decreases variations in initial angular momentum among low mass stars of a given mass. Recent observational studies indicate that the latter explanation is probably the correct one. In particular, stars in the Pleiades (age $70 \mathrm{Myr}$ ) exhibit an appreciable scatter in rotation rate at any one mass, a scatter that disappears by the age of the Hyades (age $0.6 \mathrm{Gyr}$ ) - see below.

Low mass stars first become visible to us at an age of about $1 \mathrm{Myr}$, and we call them $\mathrm{T}$ Tauri stars. Their characteristics include veiling of their spectra, excess infrared radiation, very high levels of stellar activity, etc. (see Bertout 1989 for a recent review). The veiling gradually disappears and the activity subsides, even while remaining much higher than is seen in main sequence stars. The veiling and IR excesses of $T$ Tauri stars are plausibly ascribed to the presence of disks around the $\mathrm{T}$ Tauri stars. These disks may also play a role in the angular momentum evolution of these stars - either by accretion from the disk adding angular momentum (and perhaps serving as a source of fresh lithium for the outer

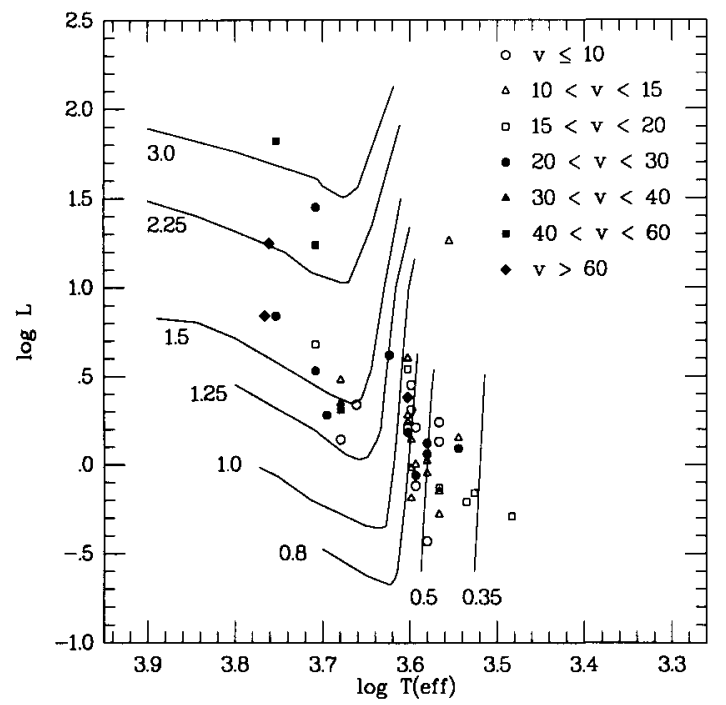

Figure 2. Observed rotational velocities ( $v \sin i$ ) among $\mathrm{T}$ Tauri stars (from Hartmann et al. 1986). Note that at and below $1 M_{\odot}$ no $v \sin i>30 \mathrm{~km} \mathrm{~s}^{-1}$ is seen, except for one star. 
envelope of the star), or by winds from a boundary layer helping extract angular momentum from the star. The disk also is the eventual source for planets (which, if our solar system is an example, can hold significantly more angular momentum than the stars which they orbit).

Among the T Tauris, the observed rotation rates at $1 M_{\odot}$ are about 6 to $30 \mathrm{~km} \mathrm{~s}^{-1}$, or 3 to $10 \Omega_{\odot}$ (Fig. 2). A number of rotation periods have been determined from variability of broad-band light, but there is a selection effect against long periods (it is difficult to obtain observational data over a period much exceeding a week or two at a time). The $\mathrm{T}$ Tauris are typically 3 times the size of the Sun, and much less centrally condensed, so there is much more angular momentum in one than in a main sequence star rotating at 3 times the solar rate. Until fairly recently it was thought that this angular momentum was lost gradually during the several tens of Myr that the star took in approaching the main sequence.

It then came as a major surprise a few years ago when some stars were discovered in the Pleiades that are rotating at as much as 100 times the solar rate yet are barely on the Zero-Age Main Sequence. These ultra-fast rotators constitute a significant fraction of the stars in the mass range in which they're seen, and are not just a few freaks (Fig. 3).

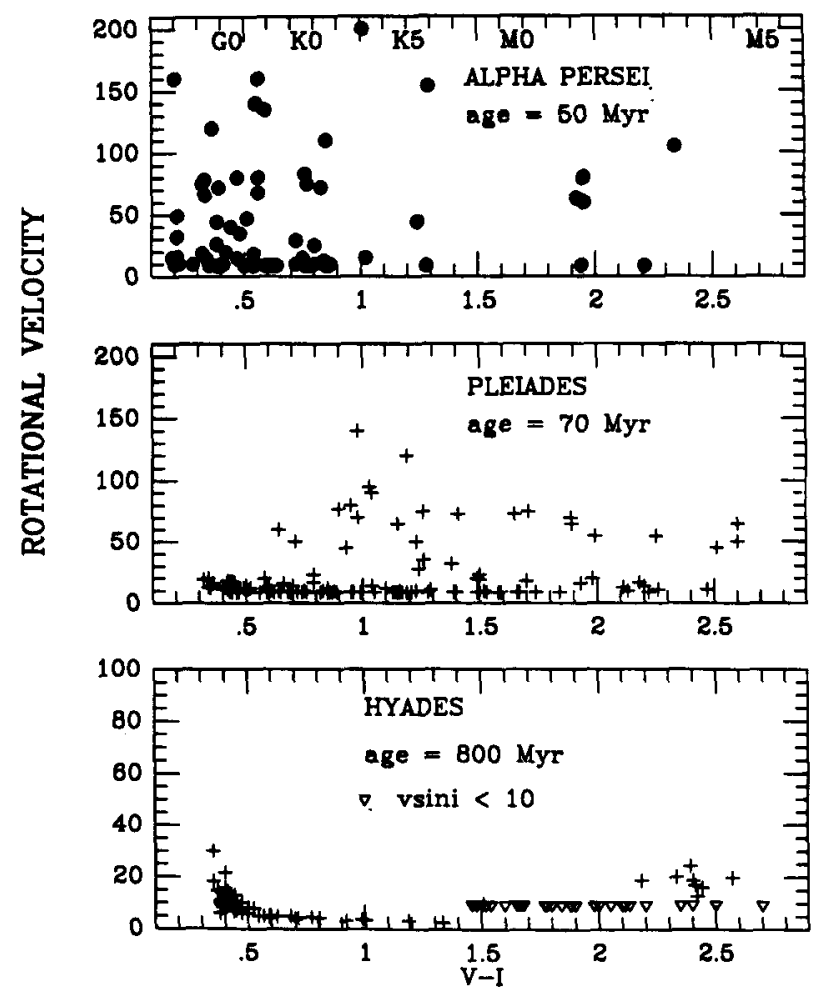

Figure 3. Distribution of rotational velocity ( $v \sin i)$ versus $(V-I)$ color for solar-type stars in the $\alpha$ Persei and Pleiades clusters (adapted from Stauffer and Hartmann 1987). The older stars of the Hyades are also shown. A $(V-I)$ color of 0.5 corresponds to about $1 M_{\odot}$, while 1.0 corresponds to about $0.8 M_{\odot}$. 
Subsequent observations of a somewhat younger cluster ( $\alpha$ Persei) showed that the presence of these unusual objects in the Pleiades was not just a fluke.

There is good evidence that the duration of this main sequence rapid rotation phase is a strong function of mass. For the youngest, well-studied cluster ( $\alpha$ Per, age $50 \mathrm{Myr}$ ), rapid rotators are found among all spectral types for low mass stars. For the slightly older Pleiades cluster ( $70 \mathrm{Myr}$ ), the $\mathrm{G}$ dwarfs are all relatively slow rotators, with rapid rotators only among the $\mathrm{K}$ and $\mathrm{M}$ dwarfs. The oldest, well-studied cluster (the Hyades, age $0.6 \mathrm{Gyr}$ ) shows rapid rotators only among the very-low-mass $M$ dwarfs. Thus the spin-down time scales must increase with decreasing stellar mass. The open cluster data allow a quantitative estimate of the spin-down time scales for stars of various masses. $1 M_{\odot}$ rapid rotators must lose a significant fraction of their angular momentum in only $\sim 20 \mathrm{Myr} ; 0.7 M_{\odot}$ stars must have a characteristic spin-down time of order $100 \mathrm{Myr}$; and $0.5 M_{\odot}$ stars apparently must have a spin-down time scale of several hundred million years.

Just recently, Stauffer et al. (1989) have observed the very young (age $30 \mathrm{Myr}$ ) cluster IC 2391, which shows a spread in rotation rates that is similar to that of the Pleiades, yet IC 2391 has a very small age spread (no more than $20 \mathrm{Myr}$ ). Stauffer et al. thus argue that an age spread cannot account for the distribution of Pleiades rotation rates at any one mass, despite the arguments of Butler et al. (1987), who saw commensurate differences in lithium abundances. Perhaps these differing rotation rates reflect the initial distribution of angular momentum among stars, as suggested by Pinsonneault, Kawaler, and Demarque (1989).

Note that it is just possible to account for the fastest rotators seen in the Pleiades and $\alpha$ Persei clusters if no substantial angular momentum loss is tolerated between the T Tauri phase and the ZAMS (Stauffer and Hartmann 1987). This is itself a problem. That is, if the spindown of these stars is so fast and efficient on the main sequence, how do they achieve such high rotation rates to begin with? Consideration of this problem has led some theorists to suggest the possibility that these stars rotate as solid bodies while approaching the ZAMS (such is necessary to transfer angular momentum from the core to the surface to explain the highest velocities), but upon reaching the ZAMS the convective envelope decouples from the core (Endal and Sofia 1981). Thus much less mass is spun down, leaving the core spinning at a high rate. Presumably the core is at least weakly coupled to the outer convective envelope so that over some longer time scale the core comes into corotation with the envelope.

Another interesting result of the study of open clusters is the evidence that the rotational evolution of low mass stars is a convergent process. That is, whereas the young clusters (viz., the Pleiades and $\alpha$ Per) show a wide range of rotational velocities at a given spectral type, this range has essentially disappeared for $\mathrm{G}$ and $\mathrm{K}$ dwarfs by the age of the Hyades (Radick et al. 1987). This is consistent with the angular momentum loss scenario usually envisioned in which the interaction of rotation and convection drive a magnetic dynamo. The resulting magnetic field can force corotation of an ionized wind beyond the stellar surface, leading to angular momentum loss. This mechanism naturally incorporates feedback.

\section{Rotation and Activity Cycles}

The Sun's 11-year activity cycle has been going on now for 300 years or more. Other old solar-type stars almost always show some kind of similar cyclic behavior (Wilson 1978), indicating that the solar cycle is a long-term phenomenon. As such, it would then seem natural that its 11-year period is an inevitable one for a star of the Sun's mass, age, and composition. 
But it doesn't work that way. These stellar activity cycles have now been observed at Mount Wilson for more than 20 years, and cycles of various periods have been seen in most of the stars observed - about 100 of them - yet no clear trends emerge. There is no correlation between, for example, the cycle period and the rotation rate, nor for the Rossby number (the ratio of the rotation period to the convective turnover time). Baliunas has pointed out in her Pierce Prize lecture that if the Sun itself were observed for only some arbitrary 20 years out of the last 300 , the range of periods one would see would be quite similar to that seen among the Mount Wilson sample. In other words, we can reasonably hope that at present the "signal" has not yet appeared from the "noise," but that it will do so in another 20 years or so. Soderblom and Baliunas (1987) give a fuller discussion of activity cycles. Figure 4 illustrates the data relating cycle period and Rossby number.
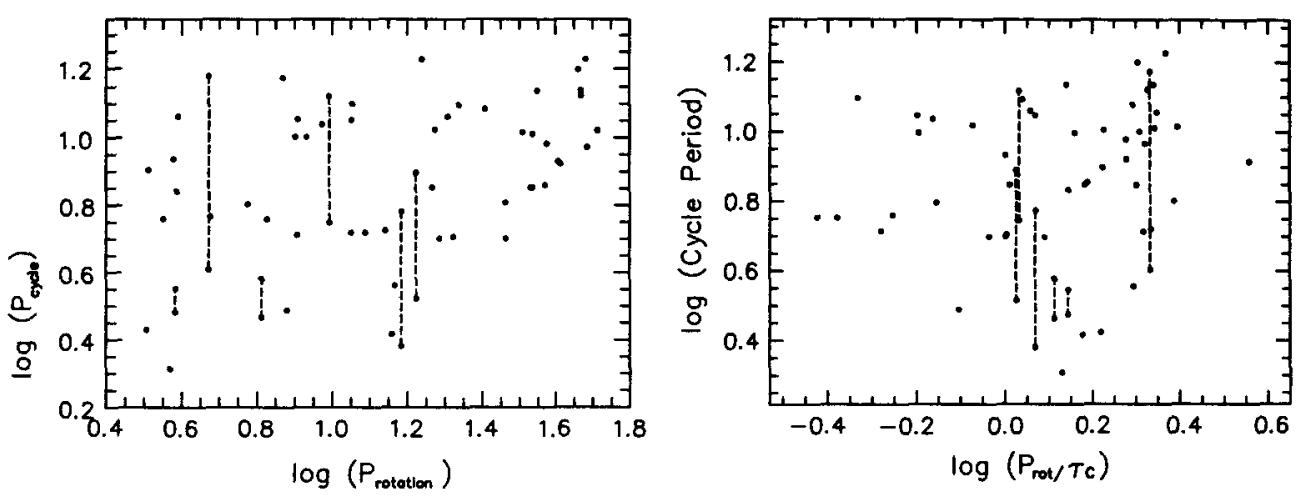

Figure 4. Observed long-term activity cycle periods (in years) versus rotation period and Rossby number (the ratio of the rotation period to the convective turnover time) for the solar-type stars observed at Mount Wilson (adapted from Soderblom and Baliunas 1987).

\section{Differential Rotation}

Relatively little is known of differential rotation in solar-type stars. For hyperactive stars like those of the RS Canum Venaticorum and BY Draconis types, there is some evidence for long-term variability that is accompanied by poleward migration of spots, in the opposite sense as is seen on the Sun (Vogt 1988). Such stars seem to commonly have enormous spots in their polar regions, which is also quite unlike the Sun.

The careful chromospheric monitoring of solar-type stars done at Mount Wilson has resulted not only in the delineation of the activity cycles just mentioned, but also for some evidence for differential rotation. That evidence (Baliunas et al. 1985) consists of changing or double peaks in the power spectrum of the spectrophotometry from season to season. Some of these cases are illustrated in Figure 5. The period changes seem unambiguous, but there is one curious feature of them: increasing activity in the star is accompanied by longer rotation periods. This is the opposite of the behavior of the Sun.

Ultimately, we must know more about differential rotation in stars like the Sun in order to better understand the dynamo mechanism and ultimately understand magnetic activity 
on the Sun itself. It is, after all, differential rotation which is instrumental in driving the dynamo, not just rotation by itself.

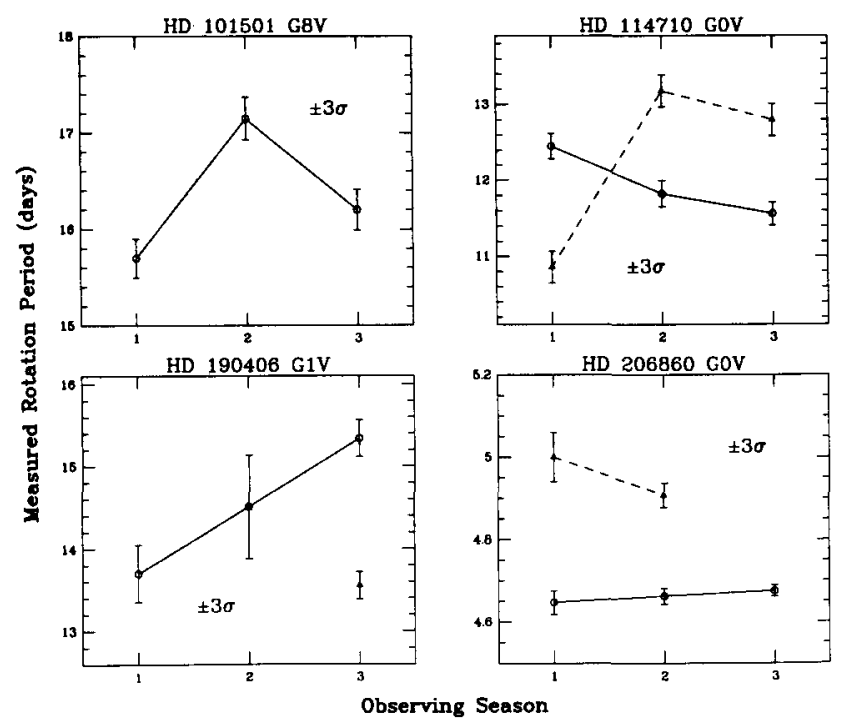

Figure 5. Rotation period changes in four solar-type stars (from Baliunas et al. 1985). In two cases the power spectrum is double-lined, indicating two periods.

\section{Does the Sun Have a Rapidly Spinning Core?}

Helioseismology is neutral on this subject, although it appears unlikely that the solar core could be rotating at a very high rate. Nevertheless, observations of $1 M_{\odot}$ that are leaving the main sequence could help one understand this problem because the convective envelopes of such stars get very deep. Thus one might see higher-than-expected rotation rates in such stars as the angular momentum in the core found its way to the surface.

Consider as an example Arcturus, which is a $\mathrm{K}$ giant star. It is single, and therefore its mass is not known with precision, but many attempts have been made to estimate its gravity spectroscopically. Those results are very consistent in indicating that Arcturus is no more massive than the Sun (the star is very close so that the radius is accurately known; it is about 20 solar radii). The problem is this: Arcturus has a measurable $v \sin i$ of about 2.5 $\mathrm{km} \mathrm{s}^{-1}$. If one collapses Arcturus back to one solar radius, the surface velocity is going to depend on how angular momentum is redistributed during post-main sequence evolution, but the star would clearly leave the main sequence with a rotation rate that is high, $\sim 50$ $\mathrm{km} \mathrm{s}^{-1}$. But the Sun, which is typical of an old solar-type star, rotates at only $2 \mathrm{~km} \mathrm{~s}^{-1}$. Where did Arcturus get this angular momentum?

There are several possible solutions to this dilemma. First, perhaps all those spectroscopic measurements are wrong and the mass is really closer to $2 M_{\odot}$. This would account for the rotation, but it seems unlikely that so many independent results could be so far off. Second, perhaps the $v \sin i$ determinations are wrong, and the real $v \sin i$ is really close to zero. The determinations that were done were done carefully, but it is conceivable that 
plausible alterations in the model atmosphere for the star could lead to a zero $v \sin i$. This is currently being investigated. Third, perhaps the observations should be taken at face value, and Arcturus has somehow managed to dip into a rapidly rotating core to achieve its present rotation rate. Fourth, the observations are $\mathrm{OK}$, but Arcturus left the main sequence with twice its current mass. Some mass loss after leaving the main sequence is not unheard of, although not quite at that level. None of these solutions is entirely satisfactory. Evolved stars with accurately known masses and in which the components do not interact are rare, but there is an interesting problem here.

\section{Rotation and Planets}

Stars more massive than about $1.5 M_{\odot}$ obey a power-law relation between their mean specific angular momenta and mass: $J / M \propto M^{-2 / 3}$. Below $1.5 M_{\odot}$, observed angular momenta fall about two orders-of-magnitude below this relation, but many have noted that if, for example, the angular momentum in the orbits of the planets is added to that of the Sun, the total falls very near the mean power-law relation. Is it possible that the primary reason for the Sun's slow rotation is that it has deposited its original angular momentum into planets?

One should first note that it is not necessary to invoke such a process to account for the slow rotation of the Sun and similar stars, for we observe the Sun to be losing angular momentum through its wind, and we observe a steady decline of rotation with age among solar-type stars (Soderblom 1983). Furthermore, no satisfactory mechanism has been put forth to transfer the angular momentum from the star to the circumstellar material. This difficulty is compounded because it appears that solar-type stars lose their disks well before reaching the ZAMS (Strom et al. 1989). It is at the ZAMS that the very high rotation rates are seen that make one consider unusual angular momentum loss mechanisms, yet deposition of angular momentum from a star into cold, solid matter would be inexplicable.

Our knowledge of the material surrounding young stars is still sufficiently poor that a mechanism involving the transfer of angular momentum to planets or a proto-planetary disk cannot be completely ruled out, but that appears a most unlikely possibility. High-spatialresolution observations of many stars of different ages by, e.g., the Hubble Space Telescope, may help.

\section{Rotation and Lithium Depletion}

The gradual depletion of lithium exhibited by stars near $1 M_{\odot}$ is surely providing some clues to processes taking place of the convective envelopes of stars. $\mathrm{Li}$ is destroyed at a temperature of about 2.4 MK, which is reached somewhat below the base of the solar convective zone. Thus some process such as convective overshooting may reasonably account for the very slow depletion of $\mathrm{Li}$, on a time scale of roughly $1 \mathrm{Gyr}$.

Observations of $\mathrm{Li}$ in solar-type stars are confusing, but the following are particularly pertinent here: First, the Pleiades cluster (age $70 \mathrm{Myr}$ ) shows a pronounced spread in rotation rates at any one mass that we have already remarked on, but there is a similar spread in the $\mathrm{Li}$. This spread grows with decreasing mass (Soderblom, Jones, and Stauffer 1990). In general, the ultra-fast rotators have about 1 dex more Li than their slowly rotating siblings, an observation that led Butler et al. (1987) to suggest that the spread in rotation and $\mathrm{Li}$ were both indicative of an age spread in the Pleiades. It is also relevant that the most 
Li-rich Pleiades stars appear to have the primordial $\mathrm{Li}$ abundance.

In the Hyades, at an age of $0.6 \mathrm{Gyr}$, things are quite different. Not only have rotation rates converged at a given mass to a uniform value, but so have the $\mathrm{Li}$ abundances (Soderblom et al. 1990). The convergence in rotation is expected because of feedback in the angular momentum loss process: stars that rotate faster should have stronger dynamos, hence larger angular momentum loss rates.

It is difficult to see how a star "knows" how to make its Li abundance converge to the "correct" value by the age of the Hyades unless there is some fairly direct connection between angular momentum loss and $\mathrm{Li}$ depletion. Such a connection is plausible if rotationallyinduced turbulence at the base of the convective envelope is the mechanism responsible for Li depletion. Pinsonneault et al. (1989) and Pinsonneault, Kawaler, and Demarque (1989) have incorporated such a mechanism in their models for the evolution of the Sun and solar-type stars. They suggest that the spread in rotation rates seen in young clusters is indicative of a spread in the angular momenta with which the stars formed, rather than representing an age spread. In other words, all the stars of the Pleiades would then be the same age but have been formed with different initial conditions. The recent observations of rotation in IC 2391 by Stauffer et al. (1989) would seem to support that hypothesis (see remarks above).

Some other conclusions about $\mathrm{Li}$ depletion can be drawn from the available data: $\mathrm{Li}$ depletion probably starts at the Zero-Age Main Sequence, not before, at least for $M \gtrsim 0.8 M_{\odot}$, because such stars in the Pleiades appear to have the primordial abundance. The situation for lower masses is not clear. Second, the depletion rate slows after the age of the Hyades. In other words, the Sun lost the greater part of its initial $\mathrm{Li}$ by an age of $0.6 \mathrm{Gyr}$, and then lost more later, but at a very slow rate.

Work now in progress should help significantly to understand how stars like the Sun have the lithium abundances that they do. Many questions arise: 1) Are all stars in the current epoch formed with the same $\mathrm{Li}$ abundance, or is there an intrinsic spread? If there is a spread, is it just between clusters, or within individual clusters? 2) Do stars alter their $\mathrm{Li}$ before reaching the ZAMS? As mentioned above, the quantities of $\mathrm{Li}$ seen in Pleiades G dwarfs suggests that the answer to this question is "no," at least for stars near $M_{\odot}$, but other observations (Strom et al. 1990) and models (Proffitt and Michaud 1989) suggest otherwise. It is possible, for example, that the high activity levels of pre-main sequence stars lead to autogenesis of $\mathrm{Li}$, which could replace some burned and lead to star-to-star differences. 3) Can a star exhibit Li variability? Very high quality observations of an active young star (Boesgaard 1988) show no evidence for variability, but this cannot be ruled out for the T Tauris. 4) What factors influence $\mathrm{Li}$ depletion and the rate at which it occurs? Clearly the star's mass is critical, but how does metallicity enter? How about rotation? 5) If these factors are specified, is there a unique $\mathrm{Li}$ abundance implied for a star, or does there remain significant uncertainty because of stochastic effects?

One of us (DS) would like to thank Cerro Tololo Interamerican Observatory for its hospitality during the completion of this review and for help with the illustrations. S. Baliunas also provided helpful information. 


\section{References}

Baliunas, S.L. et al. (1983) 'Stellar Rotation in Lower Main-Sequence Stars Measured from Time Variations in $\mathrm{H}$ and $\mathrm{K}$ Emission-Line Fluxes. II. Detailed Analysis of the 1980 Observing Season Data,' Astrophys. J., 275, 752-772.

Baliunas, S.L., et al. (1985) 'Time-Series Measurements of Chromospheric Ca II H and $\mathrm{K}$ Emission in Cool Stars and the Search for Differential Rotation,' Astrophys. J., 294, 310-325.

Bertout, C. (1989) 'T Tauri Stars: Wild as Dust,' Ann. Rev. Astron. Astrophys., 27,

Boesgaard, A.M. (1988) in G. Cayrel de Strobel and M. Spite (eds.) The Impact of Very High Signal-to-Noise Spectroscopy on Stellar Physics, IAU Symp. 132, Dordrecht, Kluwer, p. 386.

Butler, R.P., Cohen, R.D., Duncan, D.K., and Marcy, G.W. (1987) 'The Pleiades Rapid Rotators: Evidence for an Evolutionary Sequence,' Astrophys. J. Lett, 319, L19-L22.

Endal, A.S., and Sofia, S. (1981) 'Rotation in Solar-Type Stars. I. Evolutionary Models for the Spin-down of the Sun,' Astrophys. J., 243, 625-640.

Giampapa, M.S., and Sonett, C.P. (1989) 'The Sun in Time,' Tucson, Univ. of Arizona Press, in press.

Gilliland, R.L. (1987) 'The Relation of Chromospheric Activity to Convection, Rotation, and Evolution Off the Main Sequence,' Astrophys. J., 298, 286-294.

Hartmann, L.W., Hewett, R., Stahler, S., and Mathieu, R. 'Rotational and Radial Velocities of T Tauri Stars,' Astrophys. J., 309, 275-293.

Hartmann, L.W., and Noyes, R.W. (1987) 'Rotation and Magnetic Activity in Main and Sequence Stars,' Ann. Rev. Astron. Astrophys., 25, 271-301.

Kraft, R.P. (1967) 'Studies of Stellar Rotation. V. The Dependence of Rotation on Age Among Solar-Type Stars,' Astrophys. J., 150, 551-570.

Noyes, R.W., Hartmann, L.W., Baliunas, S.L., Duncan, D.K., and Vaughan, A.H. (1984) 'Rotation, Convection, and Magnetic Activity in Lower Main-Sequence Stars,' Astrophys. J., 279, 763-777.

Pinsonneault, M.H., Kawaler, S.D., and Demarque, P. (1989) 'Rotation of Low-Mass Stars: A New Probe of Stellar Evolution,' preprint.

Pinsonneault, M.H., Kalwaler, S.D., Sofia, S., and Demarque, P. (1989) 'Evolutionary Models of the Rotating Sun,' Astrophys. J., 338, 424-452.

Proffitt, C.R., and Michaud, G. (1989) 'Pre-Main Sequence Depletion of ${ }^{6} \mathrm{Li}$ and ${ }^{7} \mathrm{Li}$,' Astrophys. J., in press.

Radick, R.R., Thompson, D.T., Lockwood, G.W., Duncan, D.K., and Baggett, W.E. (1987) 'The Activity, Variability, and Rotation of Lower Main-Sequence Hyades Stars,' Astrophys. J., 321, 459-472.

Skumanich, A. (1972) 'Time Scales for Ca II Emission Decay, Rotational Braking, and Lithium Depletion,' Astrophys. J., 171, 565-567.

Soderblom, D.R. (1983) 'Rotational Studies of Late-Type Stars. II. Ages of SolarType Stars and the Rotational History of the Sun,' Astrophys. J. Suppl., 53, 1-15.

Soderblom, D.R. (1985) 'A Survey of Chromospheric Emission and Rotation Among Solar-Type Stars in the Solar Neighborhood,' Astron. J., 90, 2103-2115.

Soderblom, D.R., and Baliunas, S.L. (1987) 'The Sun Among the Stars: What the Stars Indicate About Solar Variability,' in F.R. Stephenson and A.W. Wolfendale (eds.), Secular Solar and Geomagnetic Variations in the Last 10,000 Years, NATO 
Advanced Research Workshops, Ser. C., v. 236, 25-45.

Soderblom, D.R. Jones, B.F., and Stauffer, J.R. (1990) 'The Evolution of the Lithium Abundances of Solar-Type Stars. II. The Pleiades,' Astron. J., in preparation.

Soderblom, D.R., Oey, M.S., Johnson, D.R.H., and Stone, R.P.S. (1990) 'The Evolution of the Lithium Abundances of Solar-Type Stars. I. The Hyades and Coma Berenices Clusters,' Astron. J., in press.

Stauffer, J.R., and Hartmann, L.W. (1987) 'The Distribution of Rotational Velocities for Low-Mass Stars in the Pleiades,' Astrophys. J., 318, 337-355.

Stauffer, J.R., Hartmann, L.W., Jones, B.F., and McNamara, B.R. (1989) 'Pre-MainSequence Stars in the Young Cluster IC 2391,'A Astrophys. J., 342, 285-292.

Stauffer, J.R., and Soderblom, D.R. (1989) 'The Angular Momentum History of the Sun,' in M. Giampapa and C. Sonett (eds.), The Sun in Time, Univ. of Arizona Press, in press.

Strom, K.M., Strom, S.E., Edwards, S., Cabrit, S., and Skrutskie, M.F. (1989) 'Circumstellar Material Associated with Solar-Type Pre-Main Sequence Stars: A Possible Constraint on the Timescale for Planet Building,' Astron. J., 97, 1451-1470.

Strom, K.M., Wilkin, F.P., Strom, S.E., and Seaman, R.L. (1990) 'Lithium Abundances Among Solar-Type Pre-Main Sequence Stars,' preprint.

Vogt, S.S. (1988) 'Doppler Images of Spotted Late-Type Stars,' in G. Cayrel de Strobel and M. Spite (eds.) The Impact of Very High Signal-to-Noise Spectroscopy on Stellar Physics, IAU Symp. 132, Dordrecht, Kluwer, p. 253-272.

Wilson, O.C. (1978) 'Chromospheric Variations in Main-Sequence Stars,' Astrophys. J., 226, 379-396. 